\title{
Older adults recently started on psychotropic medication: where are the symptoms?
}

\author{
Donovan T. Maust ${ }^{1,2}$, Shirley H. Chen ${ }^{3}$, Amy Benson ${ }^{4}$, Shahrzad Mavandadi ${ }^{3,4}$, Joel E. Streim ${ }^{3,4}$, \\ Suzanne DiFilippo ${ }^{3,4}$, Thomas M. Snedden ${ }^{5}$ and David W. Oslin ${ }^{3,4}$ \\ ${ }^{1}$ Department of Psychiatry, University of Michigan, Ann Arbor, MI, USA \\ ${ }^{2}$ Center for Clinical Management Research, VA Ann Arbor Healthcare System, Ann Arbor, MI, USA \\ ${ }^{3}$ Philadelphia Veterans Affairs Medical Center and the VISN 4 Mental Illness Research, Education, and Clinical Center (MIRECC), \\ Philadelphia, PA, USA \\ ${ }^{4}$ Department of Psychiatry, University of Pennsylvania, Philadelphia, PA, USA \\ ${ }^{5}$ PACE Program, Pennsylvania Department of Aging, Harrisburg, PA, USA \\ Correspondence to: D. T. Maust, MD, MS, E-mail: maustd@umich.edu
}

Objective: The objective of this study is to understand the characteristics of older adults on newly prescribed psychotropic medication with minimal psychiatric symptoms.

Methods: Naturalistic cohort study of non-institutionalized older adults in Pennsylvania participating in the Pharmaceutical Assistance Contract for the Elderly. Persons newly prescribed antidepressant or anxiolytic monotherapy or combination therapy were contacted for clinical assessment by a telephone-based behavioral health service. The initial assessment included standardized mental health screening instruments and scales including the Blessed Orientation-Memory-Concentration test, Patient Health Questionnaire-9, Generalized Anxiety Disorder-7, and Medical Outcomes Survey (SF-12). In addition, patients were asked for their understanding of the prescription indication.

Results: Of the 254 participants who met minimal symptom criteria (Patient Health Questionnaire$9<5$ and Generalized Anxiety Disorder-7 $<5$ ), women comprised slightly more of the anxiolytic compared with antidepressant monotherapy group $(88.9 \%$ vs. $76.7 \%, p=0.04)$. The most common selfreported reason for prescription of an antidepressant or anxiolytic was depression or anxiety, respectively, despite near-absence of these symptoms on clinical assessment. Comparing monotherapy to combination therapy groups, those with combination therapy were more likely to report a history of depression $(12.6 \%$ vs. $1.8 \%, p<0.001)$ and also report depression as the reason for the prescription $(40.2 \%$ vs. $21.0 \%, p<0.01)$. Conclusions: In this sample of older adults on new psychotropic medication with minimal psychiatric symptoms, there are few patient characteristics that distinguish those on antidepressant versus anxiolytic monotherapy or those on monotherapy versus combination therapy. While quality of care in late-life mental health has focused on improving detection and treatment, there should be further attention to low-symptom patients potentially receiving inappropriate pharmacotherapy. Copyright (C) 2014 John Wiley \& Sons, Ltd.

Key words: psychotropic medication; primary care; older adults

History: Received 23 January 2014; Accepted 18 July 2014; Published online 12 August 2014 in Wiley Online Library (wileyonlinelibrary.com)

DOI: 10.1002 /gps.4187

\section{Introduction}

Over the past 20 years, the detection and treatment of mental health disorders in primary care settings has been a key area of emphasis in geriatric mental health, and a variety of strategies have been developed to improve detection and treatment (Unutzer et al., 2002; Crystal et al., 2003; Oxman et al., 2003; Bruce et al., 2004; Oslin et al., 2006a). While evidence-based psychotherapies may be preferred by older adults (Landreville et al., 2001; Gum et al., 2006), pharmacotherapy is the treatment most commonly provided 
(Young et al., 2001). Concurrent with this emphasis on detection and treatment, evidence continues to accumulate regarding the potential risks of psychotropic use in older adults. The use of benzodiazepines in older adults has been associated with increased risk of falls and fractures (Wang et al., 2001; Chang et al., 2008). Although the newer antidepressants have been favored for their safety and side effect profile relative to the tricyclic antidepressants (Zwanziger and Auerbach, 1991; Rudorfer et al., 1994; Peretti et al., 2000), the selective-serotonin reuptake inhibitors have now been associated with the risk of fracture (Ginzburg and Rosero, 2009) and adverse cognitive effects (Culang et al., 2009; Sneed et al., 2010), while mirtazapine and venlafaxine have been associated with stroke and fracture (Coupland et al., 2011a, 2011b). Most psychotropic medications are listed as at least potentially inappropriate in the most recent iteration of the American Geriatrics Society Beers Criteria (American Geriatrics Society, 2012).

The emphasis on treatment of mental health disorders in primary care paired with the growing attention to risks of psychotropic use has led to work exploring the potentially inappropriate use of psychotropic medication in primary care, such as prescribing in the absence of a psychiatric disorder. Survey and administrative data have documented the significant use of psychotropic medication in the absence of psychiatric diagnoses or mental health complaints (Pagura et al., 2011; Wiechers et al., 2013), which appears to be even more likely in older adults (Wiechers et al., 2014; Maust et al., 2014). While these findings are important, a limitation of these analyses is that they do not use patient-level clinical data. Although a diagnosis of depression may not have been recorded, the patient in fact may have been depressed and the clinician responded appropriately.

The Behavioral Health Laboratory (BHL) is an evidence-based clinical service delivered by telephone to help identify and manage the mental health needs of patients (Oslin et al., 2006b). Since 2008, the Commonwealth of Pennsylvania's Pharmaceutical Assistance Contract for the Elderly (PACE) has contracted with BHL to provide clinical services to older adults in Pennsylvania who have been newly prescribed an antidepressant, anxiolytic, or antipsychotic. Based on contact with older adults across the state, we were the first with patient-level clinical information to describe psychotropic use in minimally symptomatic older adults (Maust et al., 2011).

On the basis of these baseline analyses, we decided to prospectively identify and follow the cohort of older adults with a minimal level of psychiatric symptoms.
This minimal symptom cohort was prospectively defined as meeting the following three criteria: minimal symptoms of depression (Patient Health Questionnaire-9 [PHQ-9] < 5), minimal symptoms of anxiety (Generalized Anxiety Disorder-7 [GAD-7] $<5$ ), and a negative screen for any other Axis I psychiatric disorders. Having provided the BHL clinical service over several additional years, the aims of these analyses were twofold. First, we wanted to see whether our initial findings were replicable. If so, we then wanted to gain further understanding of the use of psychotropic medication in minimally symptomatic older adults. In order to do so, we examined if and how minimally symptomatic older adults prescribed antidepressants versus anxiolytics compared on factors such as sociodemographics, treatment history, and current symptoms. Next, we compared patients for whom the index medication was the only psychotropic medication with those already receiving a psychotropic medication. We hypothesized that those on combination psychotropic regimens would be more likely to report previous mental health treatment and more likely to endorse worse overall physical and emotional health.

\section{Methods}

These analyses are based on an ongoing clinical program. The data collection methods that follow are similar to those described in previous publications (Maust et al., 2011, 2013a).

\section{Sample}

This cohort is drawn from low-income older adults in the state of Pennsylvania who participate in the state's PACE, a program of the Pennsylvania Department of Aging that provides comprehensive prescription coverage for low-income residents 65 years or older. Participants were contacted for clinical assessment by the BHL as part of a clinical contract with the PACE program. PACE enrollees eligible for contact were adults in a non-institutionalized setting who filled at least one new prescription for an antidepressant, antipsychotic, and/or anxiolytic between August 2010 and February 2013. A prescription was considered new if no prior pharmacy claim to PACE had been made for that medication class in a preceding 9-month period of continuous enrollment. Enrollees were randomly selected for the BHL clinical assessment every week during this period from all claims submitted to PACE for newly written prescriptions, stratified by county (Philadelphia area, Allegheny area, 
and other). Stratification by county was performed to ensure that the sample was not dominated by enrollees in the state's large urban centers. Prescriptions written by providers identified by PACE as psychiatrists were excluded from this analysis.

Enrollees sampled by PACE who agreed to participate in the BHL clinical assessment underwent an initial baseline telephone assessment that was administered by staff BHL health technicians (not otherwise involved in the enrollees' outpatient care). These analyses here are limited to claims for antidepressants and anxiolytics, as these medications may be prescribed for similar complaints.

\section{Measures}

The baseline interview included sociodemographics, and standardized scales administered included the following:

(1) Blessed Orientation-Memory-Concentration (BOMC) test, range 0 to 28 where $>10$ suggests cognitive impairment (Katzman et al., 1983);

(2) MINI International Neuropsychiatric Interview (includes psychosis, mania, GAD, Panic disorder, and alcohol abuse/dependence modules), a structured diagnostic interview for DSM-IV disorders (Sheehan et al., 1998);

(3) PHQ-9, a brief self-report depression severity measure based on self-report where scores of 5, 10,15 , and 20 represent mild, moderate, moderately severe, and severe depression, respectively (Kroenke et al., 2001);

(4) GAD-7, a brief self-report anxiety measure where scores of 5, 10, and 15 reflect mild, moderate, and severe anxiety, respectively (Kroenke et al., 2007);

(5) Medical Outcomes Survey (SF-12), a set of 12 items from the SF-36 Health Survey yielding physical and mental summary measures where the adult population average is 50 on both the physical and mental summary measures (Ware et al., 1996).

In addition, interviewees were asked about past episodes of depression significant enough to interfere with social or occupation function (Oslin et al., 2006b). Finally, interviewees were asked for their understanding of the reason for the index medication (the medication that prompted contact from the BHL). Their response was recorded as free text, which the health technician conducting the interview also categorized it on the basis of a brief list of forced-choice options.
Interviews began with the BOMC; for those who scored $\geq 14$, the interview with the PACE enrollee was terminated, and permission was sought to speak with a caregiver/loved one. This analysis only includes data from participant interviews and does not include the caregiver interview data. After completing the clinical interview, participants provided oral consent to allow the use of clinical data for research purposes in accordance with a University of Pennsylvania Institutional Review Board-approved protocol.

Analytic plan

The analyses here are limited to those who, based on the initial clinical contact, met the criteria for the minimal symptom cohort (PHQ-9 $<5$, GAD-7 $<5$, and negative screens on MINI modules). The first analyses compared those on antidepressant or anxiolytic monotherapy; the second compared those on any monotherapy versus combination therapy. Descriptive univariate analyses included calculating means and standard deviations for continuous outcomes and calculating frequencies and percentages for categorical outcomes. To compare sociodemographic/background characteristics and BHL mental health assessment outcomes across medication class, we employed Wilcoxon two-sample and chi-square tests for continuous and categorical variables, respectively. Categorical variables with small cell counts $(<5)$ were compared using Fisher's exact test. Lastly, we performed a sensitivity analysis comparing symptom levels within treatment groups by time to contact. All analyses were two-tailed, with a $p$-value $\leq 0.05$ denoting statistical significance. Analyses were conducted using SAS version 9.3 (SAS Institute Inc., Cary, NC, USA).

\section{Results}

Contact was attempted for 4907 potential participants, of whom $1313(26.8 \%)$ were able and willing to complete the full core interview. One thousand three hundred participants $(26.5 \%)$ were unable to be reached despite multiple attempts (the contact information available to BHL was not up to date, in many cases), 1547 (31.5\%) refused, and 409 (8.3\%) were unable to complete an assessment for reasons such as a language barrier or communication impediment (such as hearing impairment). Of the 1313 that agreed to participate, $552(42.0 \%)$ met the criteria for the minimal symptom cohort. One hundred twenty-six $(9.6 \%)$ had $\mathrm{BOMC} \geq 14$, so only caregivers completed an interview, whereas seven $(0.5 \%)$ did not consent to allow their information to be used for 
research purposes. Overall, the mean time to contact was 16.6 days (standard deviation [SD] 9.8). The results that follow are for the 254 participants (19.3\%) who: completed the core interview, met the criteria for the minimal symptom cohort, and confirmed that the index medication was a newly prescribed medication.

Results from the monotherapy group are presented in Tables 1 and 2. For example, the average PHQ-9 score for enrollees newly prescribed an antidepressant was 1.9 (SD 1.4), whereas the average GAD-7 for enrollees newly prescribed an anxiolytic was 1.0 (SD 1.2). The only significant difference between the antidepressant and anxiolytic groups was gender, with the anxiolytic users even more likely to be female than in the antidepressant group $\left(X^{2}(1)=4.29, p=0.04\right)$. Patients' self-report of the reason they were prescribed the new antidepressant or anxiolytic (Table 2) generally follow medication class, where those prescribed antidepressants were most likely to endorse symptoms categorized as depressive (Fisher's exact, $p<0.001$ ) and those on anxiolytics were most likely to report anxiety $\left(X^{2}(1)=25.26, p<0.001\right)$. Time to contact was 16.2 days (SD 9.8) and 17.5 days (SD 8.8) for the antidepressant and anxiolytic groups, respectively $(Z=0.00, p=1.00)$.

Results comparing the psychotropic combination to monotherapy groups are presented in Tables 3 and 4 . The only demographic or clinical characteristic on which they varied (Table 3) was past history of depression, which the combination group was more likely to endorse $(p<0.001)$. The combination group was more likely to endorse depressive-type symptoms as the reason for prescription of the new medication $\left(X^{2}(1)=10.64, p<0.01\right)$. Otherwise, the monotherapy and combination therapy groups did not differ significantly in self-reported reason for psychotropic prescription. Time to contact was 16.8 days (SD 9.8) and 16.1 days (SD 9.9) for the antidepressant and anxiolytic groups, respectively $(Z=-0.48, p=0.64)$.

Lastly, we used the median time to contact (14 days) to split each of the four groups (antidepressant and anxiolytic monotherapy; monotherapy and combination therapy) and compare symptom burden. There was no significant difference by speed of contact for any of the treatment groups. For example, when comparing depression symptoms of those on a new antidepressant that were contacted $\leq 14$ and $>14$ days from starting the medication, there was no statistically significant difference in PHQ-9 (1.88 [SD 1.41] vs. 1.85 [SD 1.39], respectively; $Z=-0.14, p=0.89$ ).

\section{Discussion}

In 2011, we published the first analyses with patientlevel clinical information describing the use of new psychotropic medication in older adults despite minimal psychiatric symptoms (Maust et al., 2011). In those analyses, $48 \%$ of PACE enrollees on newly prescribed psychotropic medication did not meet screening criteria

Table 1 Sociodemographic and clinical characteristics of Pharmaceutical Assistance Contract for the Elderly participants on antidepressant or anxiolytic monotherapy

\begin{tabular}{|c|c|c|c|c|}
\hline Characteristic & $\begin{array}{l}\text { Monotherapy } \\
n=167\end{array}$ & $\begin{array}{l}\text { Antidepressant } \\
n=86(51.5 \%)\end{array}$ & $\begin{array}{c}\text { Anxiolytic } \\
n=81(48.5 \%)\end{array}$ & Test, $p$-value \\
\hline Age (mean, SD) & $78.6(6.6)$ & $78.5(6.6)$ & $78.6(6.6)$ & $Z=0.00, p=1.00$ \\
\hline Female & $138(82.6)$ & $66(76.7)$ & $72(88.9)$ & $X^{2}(1)=4.29, p=0.04$ \\
\hline White & $156(93.3)$ & 79 (91.9) & $77(95.1)$ & $\begin{aligned} p^{*} & =0.54\end{aligned}$ \\
\hline Financial status (has at least "enough to get by") & $157(94.0)$ & $80(93.0)$ & $77(95.1)$ & $p^{*}=0.75$ \\
\hline Married & $47(28.1)$ & $24(27.9)$ & $23(28.4)$ & $X^{2}(1)=0.00, p=0.94$ \\
\hline $\begin{array}{l}\text { Number of unique medications } 6 \text { months prior to } \\
\text { index medication }\end{array}$ & $7.5(4.1)$ & $7.4(3.7)$ & $7.6(4.5)$ & $Z=-0.27, p=0.78$ \\
\hline Number of unique mediations 6 months after index medication & $7.0(4.1)$ & $7.2(4.2)$ & $6.8(4.1)$ & $Z=-0.63, p=0.53$ \\
\hline $\begin{array}{l}\text { Overall general functioning (SF-12): physical } \\
\text { component score (mean, SD) }\end{array}$ & $44.6(10.2)$ & $43.8(10.7)$ & $45.4(9.7)$ & $Z=0.99, p=0.33$ \\
\hline $\begin{array}{l}\text { Overall general functioning (SF-12): mental } \\
\text { component score (mean, SD) }\end{array}$ & $56.7(5.7)$ & $56.0(5.8)$ & $57.4(5.5)$ & $Z=1.54, p=0.13$ \\
\hline In mental health care in past 2 years & $2(1.2)$ & $0(0)$ & $2(2.5)$ & $p^{*}=0.23$ \\
\hline In mental health care in past 3 months & $1(0.6)$ & $0(0)$ & $1(1.2)$ & $p^{*}=0.49$ \\
\hline Past history of depression & $3(1.8)$ & $2(2.3)$ & $1(1.2)$ & $p^{*}=1.00$ \\
\hline Cognitive function (blessed score) (mean, SD) & $5.0(3.8)$ & $5.1(4.0)$ & $4.9(3.4)$ & $Z=-0.09, p=0.93$ \\
\hline PHQ-9 score (mean, SD) & $1.9(1.4)$ & $1.9(1.4)$ & $1.8(1.4)$ & $Z=-0.35, p=0.73$ \\
\hline GAD-7 score (mean, SD) & $0.8(1.1)$ & $0.7(1.0)$ & $1.0(1.2)$ & $Z=1.32, p=0.19$ \\
\hline
\end{tabular}

PHQ-9, Patient Health Questionnaire-9; GAD-7, Generalized Anxiety Disorder-7; SD, standard deviation.

*Fisher's exact test. 
Table 2 Self-report of prescription indication for participants on antidepressant or anxiolytic monotherapy

\begin{tabular}{|c|c|c|c|c|}
\hline Self-reported reason & $\begin{array}{c}\text { Monotherapy } \\
n=167\end{array}$ & $\begin{array}{c}\text { Antidepressant } \\
n=86(51.5 \%)\end{array}$ & $\begin{array}{c}\text { Anxiolytic } \\
n=81(48.5 \%)\end{array}$ & $X^{2}, p$-value ${ }^{a}$ \\
\hline Anxiety (stress, tension, worry, and nervousness) & $82(49.1)$ & $26(30.2)$ & $56(69.1)$ & $25.26, p<0.001$ \\
\hline Depression (low mood and lack of interest) & $35(21.0)$ & $32(37.2)$ & $3(3.7)$ & $p^{*}<0.001$ \\
\hline Stressful life event, adjustment, and grief & $1(0.6)$ & $0(0)$ & $1(1.2)$ & $p^{\star}=0.49$ \\
\hline Sleep difficulties & $33(19.8)$ & $11(12.8)$ & $22(27.2)$ & $5.43, p=0.02$ \\
\hline Pain & $16(9.6)$ & $12(14.0)$ & $4(4.9)$ & $p^{*}=0.06$ \\
\hline Physical/somatic condition & $16(9.6)$ & $7(8.1)$ & $9(11.1)$ & $0.43, p=0.51$ \\
\hline Irritability (agitation amd anger) & $0(0)$ & $0(0)$ & $0(0)$ & $\mathrm{n} / \mathrm{a}$ \\
\hline Cognitive/memory difficulties & $2(1.2)$ & $2(2.3)$ & $0(0)$ & $p^{*}=0.50$ \\
\hline Miscellaneous & $12(7.2)$ & $6(7.0)$ & $6(7.4)$ & $0.01, p=0.91$ \\
\hline Reason unknown (e.g. " "don’t know") & $3(1.8)$ & $3(3.5)$ & $0(0)$ & $p^{\star}=0.25$ \\
\hline
\end{tabular}

ad.f. $=1$ for all comparisons.

*Fisher's exact test.

Table 3 Sociodemographic and clinical characteristics of Pharmaceutical Assistance Contract for the Elderly participants on psychotropic monotherapy versus combination therapy

\begin{tabular}{|c|c|c|c|c|}
\hline Characteristic & $\begin{array}{l}\text { All interviewees } \\
\qquad n=254\end{array}$ & $\begin{array}{l}\text { Monotherapy } \\
n=167(65.7 \%)\end{array}$ & $\begin{array}{l}\text { Combination } \\
n=87(34.3 \%)\end{array}$ & Test, $p$-value \\
\hline Age (mean, SD) & $78.5(6.4)$ & $78.6(6.6)$ & $78.3(6.1)$ & $Z=-0.25, p=0.81$ \\
\hline Female & 211 (83.1) & $138(82.6)$ & 73 (83.9) & $X^{2}(1)=0.07, p=0.80$ \\
\hline White & 239 (94.1) & 156 (93.4) & $83(95.4)$ & $p^{\star}=0.60$ \\
\hline Financial Status (has at least "enough to get by") & $235(92.5)$ & $157(94.0)$ & $78(89.7)$ & $X^{2}(1)=1.57, p=0.21$ \\
\hline Married & $68(26.8)$ & $47(28.1)$ & $21(24.1)$ & $X^{2}(1)=0.47, p=0.49$ \\
\hline $\begin{array}{l}\text { Number of unique medications, } 6 \text { months prior to } \\
\text { index medication }\end{array}$ & $7.8(4.0)$ & $7.5(4.1)$ & $8.5(3.9)$ & $Z=2.13, p=0.03$ \\
\hline $\begin{array}{l}\text { Number of unique mediations, } 6 \text { months after } \\
\text { index medication }\end{array}$ & $7.3(4.1)$ & $7.0(4.1)$ & $8.0(4.1)$ & $Z=1.88, p=0.06$ \\
\hline $\begin{array}{l}\text { Overall general functioning (SF-12): physical component } \\
\text { score (mean, SD) }\end{array}$ & $44.3(10.6)$ & $44.6(10.2)$ & $43.8(11.3)$ & $Z=-0.42, p=0.67$ \\
\hline $\begin{array}{l}\text { Overall general functioning (SF-12): mental component } \\
\text { score (mean, SD) }\end{array}$ & $56.5(5.9)$ & $56.7(5.7)$ & $56.1(6.2)$ & $Z=-.45, p=0.66$ \\
\hline In mental health care in past 2 years & $6(2.4)$ & $2(1.2)$ & $4(4.6)$ & $p^{\star}=0.19$ \\
\hline In mental health care in past 3 months & $3(1.2)$ & $1(0.6)$ & $2(2.3)$ & $p^{*}=0.27$ \\
\hline Past history of depression & $14(5.5)$ & $3(1.8)$ & $11(12.6)$ & $p^{*}<0.001$ \\
\hline Cognitive function (blessed score) (mean, SD) & 4.9 (3.8) & $5.0(3.8)$ & $4.7(3.8)$ & $Z=-0.60, p=0.55$ \\
\hline PHQ-9 score (mean, SD) & $1.9(1.4)$ & $1.9(1.4)$ & $1.9(1.4)$ & $Z=0.41, p=0.68$ \\
\hline GAD-7 score (mean, SD) & $0.9(1.2)$ & $0.8(1.1)$ & $1.1(1.3)$ & $Z=1.85, p=0.07$ \\
\hline
\end{tabular}

PHQ-9, Patient Health Questionnaire-9; GAD-7, Generalized Anxiety Disorder-7; SD, standard deviation.

${ }^{\wedge}$ This monotherapy group is the 86 antidepressant users and 81 anxiolytic users presented in Tables 1 and 2.

*Fisher's exact test.

for any psychiatric disorder. Therefore, the first aim of these analyses was to replicate this surprising finding. While slightly lower, of those PACE enrollees that agreed to participate, we found that $42 \%$ of those on a newly prescribed psychotropic medication were essentially asymptomatic. We then conducted analyses considering those on psychotropic monotherapy versus combination therapy in order to gain some additional understanding of the phenomenology of prescribing to these minimally symptomatic older adults. Ultimately, we found very little to distinguish the groups.

Monotherapy antidepressant and anxiolytic users reported being prescribed with the medication for reasons consistent with depression and anxiety, respectively. However, these analyses focus on a cohort that, by definition, endorsed almost no symptoms consistent with syndromal depressive or anxiety disorders in their clinical assessment. The average PHQ-9 score for the antidepressant users was just 1.9 (SD 1.4), where 10 is the screening cutoff for major depressive disorder (Kroenke et al., 2001). Compared with the monotherapy group, the combination therapy patients were more likely to report a past history of depression as well as reporting depression as the indication for the prescription. However, the combination did not endorse any more mental health 
Table 4 Self-report of prescription indication for participants on psychotropic monotherapy versus combination therapy

\begin{tabular}{|c|c|c|c|c|}
\hline Self-reported reason & $\begin{array}{c}\text { All interviewees } \\
n=254\end{array}$ & $\begin{array}{l}\text { Monotherapy^ } \\
n=167(65.7 \%)\end{array}$ & $\begin{array}{c}\text { Combination } \\
n=87(34.3 \%)\end{array}$ & $X^{2}, p$-value ${ }^{a}$ \\
\hline Anxiety (stress, tension, worry, and nervousness) & 119 (46.9) & $82(49.1)$ & $37(42.5)$ & $0.99, p=0.32$ \\
\hline Depression (low mood and lack of interest) & $70(27.6)$ & $35(21.0)$ & 35 (40.2) & $10.64, p<0.01$ \\
\hline Stressful life event, adjustment, and grief & $2(0.8)$ & $1(0.6)$ & $1(1.1)$ & $p^{*}=1.00$ \\
\hline Sleep difficulties & $50(19.7)$ & $33(19.8)$ & $17(19.5)$ & $0.00, p=0.97$ \\
\hline Pain & $21(8.3)$ & $16(9.6)$ & $5(5.7)$ & $p^{\star}=0.35$ \\
\hline Physical/somatic condition & $21(8.3)$ & $16(9.6)$ & $5(5.7)$ & $p^{*}=0.35$ \\
\hline Irritability (agitation and anger) & $0(0)$ & $0(0)$ & $0(0)$ & $\mathrm{n} / \mathrm{a}$ \\
\hline Cognitive/memory difficulties & $2(0.8)$ & $2(1.2)$ & $0(0)$ & $p^{\star}=0.55$ \\
\hline Miscellaneous & $16(6.3)$ & $12(7.2)$ & $4(4.6)$ & $p^{*}=0.59$ \\
\hline Reason unknown (e.g., "don’t know") & $5(2.0)$ & $3(1.8)$ & $2(2.3)$ & $p^{*}=1.00$ \\
\hline
\end{tabular}

${ }^{\wedge}$ This monotherapy group is the 86 antidepressant users and 81 anxiolytic users presented in Tables 1 and 2.

ad.f. $=1$ for all comparisons.

*Fisher's exact test.

care utilization nor was their depression or anxiety symptom burden any higher.

While we are not seeking to describe the prevalence of low-symptom prescribing, our patient-level findings are consistent with recent secondary analyses of psychotropic use in the absence of a psychiatric diagnosis. Using a private insurance claims database, Wiechers et al. recently reported that $58 \%$ of individuals prescribed with a psychotropic medication in 2009 had no psychiatric diagnosis (Wiechers et al., 2014). In data from the Veterans Health Administration, $30 \%$ of the patients had no psychiatric diagnosis. This was most common in older adults, where approximately $60 \%$ of patients over 65 in non-mental health practices did not have a psychiatric diagnosis (Wiechers et al., 2013). Analyses of the National Ambulatory Medical Care Survey have also found that prescribing in the absence of a psychotropic diagnosis is more pronounced in older adults (Maust et al., 2014)

A key limitation of these analyses is that the baseline telephone clinical assessment is not performed at the same time as the prescribing clinician's assessment of the patient, so we do not capture the clinical presentation that prompts the prescription. Given time-to-onset of psychotropic response, particularly of antidepressants in older adults, it is unexpected that participants would essentially be symptom-free by the time of BHL contact, although benzodiazepine response is more rapid. Our sensitivity analyses do not suggest that the symptom burden varies by the speed of contact. A second limitation is that we do not know to what extent the enrollees we contact are representative of those who we are unable to contact or who are unwilling to participate. Those unwilling to participate may have had a higher symptom burden; on the other hand, those that could not be contacted may have been more active and therefore more difficult to contact. It is unclear in what way this non-response may bias our results relative to the true prevalence of low-symptom prescribing. Lastly, it may be that the PHQ-9 and GAD-7 are not fully capturing the actual burden of psychiatric symptoms. However, the SF-12 mental component score is above 50 for all monotherapy and combination therapy groups, which means these enrollees all reported above-average mental well-being (Ware et al., 1996), consistent with their low PHQ-9 and GAD-7 scores.

\section{Conclusion}

Going forward, older patients in primary care practices may be more receptive to psychotropic medication than the current population of older adults, as opinions toward psychotropic medications generally become more favorable (Mojtabai, 2009). As prescribing increases, potentially inappropriate use of psychotropic medication in the absence of significant psychiatric symptoms will likely become more common. Although the emphasis on under-diagnosis and under-treatment is important, providers should also recognize that medications are not benign, and there are risks associated with poorly targeted treatment. Going forward, it will be important to use patient-level clinical data in other populations and care systems to replicate our finding of psychotropic use in these minimally symptomatic older adults. In addition, as older adults generally seek care for mental health needs in primary care settings, it is important that primary care providers receive more training in the diagnosis and treatment of mental health disorders 
(Institute of Medicine, 2012). As implementation of mental health parity and population-based care continues, it will be essential to implement models of collaborative care for late-life mental health disorders that can support primary care providers and increase the sensitivity and specificity of treatment efforts (Bao et al., 2013; Katon and Unutzer, 2013; Maust et al., 2013b).

\section{Conflict of interest}

None declared.

\section{Key points}

- Psychotropic use occurs among older adults in the community with minimal symptoms.

- There are no clear associations between clinical profile and the use of psychotropic monotherapy or combination therapy among low-symptom older adults.

\section{Acknowledgements}

This work was funded by the PACE Program of the Pennsylvania Department of Aging. Mr Snedden directs the PACE Program. The authors have no further disclosures to report.

\section{References}

American Geriatrics Society 2012 Beers Criteria Update Expert Panel. 2012. American geriatrics society updated beers criteria for potentially inappropriate medication use in older adults. J Am Geriatr Soc 60: 616-631.

Bao Y, Casalino LP, Pincus HA. 2013. Behavioral health and health care reform models: patient-centered medical home, health home, and accountable care organization. J Behav Health Serv Res 40: 121-132.

Bruce ML, Ten Have TR, Reynolds CF, et al. 2004. Reducing suicidal ideation and depressive symptoms in depressed older primary care patients: a randomized controlled trial. JAMA 291: 1081-1091.

Chang CM, Wu EC, Chang IS, Lin KM. 2008. Benzodiazepine and risk of hip fractures in older people: a nested case-control study in Taiwan. Am J Geriatr Psychiatry 16: 686-692.

Coupland C, Dhiman P, Morriss R, et al. 2011a. Antidepressant use and risk of adverse outcomes in older people: population based cohort study. BMJ 343: d4551.

Coupland CA, Dhiman P, Barton G, et al. 2011b. A study of the safety and harms of antidepressant drugs for older people: a cohort study using a large primary care database. Health Technol Assess 15: 1-202.

Crystal S, Sambamoorthi U, Walkup JT, Akıncıgil A. 2003. Diagnosis and treatment of depression in the elderly medicare population: predictors, disparities, and trends. J Am Geriatr Soc 51: 1718-1728.

Culang ME, Sneed JR, Keilp JG, et al. 2009. Change in cognitive functioning following acute antidepressant treatment in late-life depression. Am J Geriatr Psychiatry 17: $881-888$.
Ginzburg R, Rosero E. 2009. Risk of fractures with selective serotonin-reuptake inhibitors or tricyclic antidepressants. Ann Pharmacother 43: 98-103.

Gum AM, Areán PA, Hunkeler E, et al. 2006. Depression treatment preferences in older primary care patients. Gerontologist 46: 14-22.

Institute of Medicine. 2012. The Mental Health and Substance Use Workforce for Older Adults: In Whose Hands? National Academies Press: Washington, DC.

Katon WJ, Unutzer J. 2013. Health reform and the affordable care act: the importance of mental health treatment to achieving the triple aim. J Psychosom Res 74: 533-537.

Katzman R, Brown T, Fuld P, et al. 1983. Validation of a short orientation-memoryconcentration test of cognitive impairment. Am J Psychiatry 140: 734-739.

Kroenke K, Spitzer RL, Williams JB. 2001. The PHQ-9: validity of a brief depression severity measure. J Gen Intern Med 16: 606-613.

Kroenke K, Spitzer RL, Williams JB, Monahan PO, Lowe B. 2007. Anxiety disorders in primary care: prevalence, impairment, comorbidity, and detection. Ann Intern Med 146: 317-325.

Landreville P, Landry J, Baillargeon L, Guérette A, Matteau É. 2001. Older adults' acceptance of psychological and pharmacological treatments for depression. $J$ Gerontol B Psychol Sci Soc Sci 56: 285-291.

Maust DT, Mavandadi S, Eakin A, et al. 2011. Telephone-based behavioral health assessment for older adults starting a new psychiatric medication. Am J Geriatr Psychiatry 19: 851-858.

Maust DT, Mavandadi S, Benson A, et al. 2013a. Telephone-based care management for older adults initiated on psychotropic medication. Int $J$ Geriatr Psychiatry 28: $410-416$.

Maust DT, Oslin DW, Marcus SC. 2013b. Mental health care in the accountable care organization. Psychiatr Serv 64: 908-910.

Maust DT, Oslin D, Marcus SC. 2014. Effect of Age on the profile of psychotropic users: results from the 2010 National Ambulatory Medical Care Survey. J Am Geriatr Soc 62: 358-364.

Mojtabai R. 2009. Americans' attitudes toward psychiatric medications: 1998-2006. Psychiatr Serv 60: 1015-1023.

Oslin DW, Grantham S, Coakley E, et al. 2006a. PRISM-E: comparison of integrated care and enhanced specialty referral in managing at-risk alcohol use. Psychiatr Serv 57: 954-958.

Oslin DW, Ross J, Sayers S, et al. 2006b. Screening, assessment, and management of depression in VA primary care clinics. The Behavioral Health Laboratory. J Gen Intern Med 21: 46-50.

Oxman TE, Dietrich AJ, Schulberg HC. 2003. The depression care manager and mental health specialist as collaborators within primary care. Am J Geriatr Psychiatr 11: 507-516.

Pagura J, Katz LY, Mojtabai R, et al. 2011. Antidepressant use in the absence of common mental disorders in the general population. J Clin Psychiatry 72: 494-501.

Peretti S, Judge R, Hindmarch I. 2000. Safety and tolerability considerations: tricyclic antidepressants vs. selective serotonin reuptake inhibitors. Acta Psychiatr Scand Suppl 403: 17-25.

Rudorfer MV, Manji HK, Potter WZ. 1994. Comparative tolerability profiles of the newer versus older antidepressants. Drug Saf 10: 18-46.

Sheehan DV, Lecrubier Y, Sheehan KH, et al. 1998. The Mini-International Neuropsychiatric Interview (M.I.N.I.): the development and validation of a structured diagnostic psychiatric interview for DSM-IV and ICD-10. J Clin Psychiatry 59: 22-33.

Sneed JR, Culang ME, Keilp JG, et al. 2010. Antidepressant medication and executive dysfunction: a deleterious interaction in late-life depression. Am J Geriatr Psychiatry 18: 128-135.

Unutzer J, Katon W, Callahan CM, et al. 2002. Collaborative care management of late-life depression in the primary care setting: a randomized controlled trial. JAMA 288: 2836-2845.

Wang PS, Bohn RL, Glynn RJ, Mogun H, Avorn J. 2001. Hazardous benzodiazepine regimens in the elderly: effects of half-life, dosage, and duration on risk of hip fracture. Am J Psychiatry 158: 892-898.

Ware J Jr, Kosinski M, Keller SD. 1996. A 12-item short-form health survey: construction of scales and preliminary tests of reliability and validity. Med Care 34: 220-233.

Wiechers IR, Leslie DL, Rosenheck RA. 2013. Prescribing of psychotropic medications to patients without a psychiatric diagnosis. Psychiatr Serv 64: 1243-1248.

Wiechers IR, Kirwin PD, Rosenheck RA. 2014. Increased risk among older veterans of prescribing psychotropic medication in the absence of psychiatric diagnoses. Am J Geriatr Psychiatry 22: 531-539.

Young AS, Klap R, Sherbourne CD, Wells KB. 2001. The quality of care for depressive and anxiety disorders in the United States. Arch Gen Psychiatry 58: 55-61.

Zwanziger J, Auerbach RR. 1991. Evaluating PPO performance using prior expenditure data. Med Care 29: 142-151. 